\title{
Prevalence of thyroid disorders in antenatal women and its impact on maternal and foetal outcome
}

\author{
Reshmi Ramachandran $^{1 *}$, Lakshmi Mohan ${ }^{2}$, Merin Sara Jose ${ }^{3}$ \\ ${ }^{\mathbf{1}}$ Assistant Professor, ${ }^{2}$ Assistant Surgeon, ${ }^{3}$ Senior Resident, ${ }^{1,3}$ Dept. of Community Medicine, ${ }^{1,3}$ Government Medical College, Ernakulam, \\ Kerala, ${ }^{2}$ UHTC Ambalapuzha, Alappuzha, Kerala, India \\ *Corresponding Author: Reshmi Ramachandran \\ Email: reshmirhiju@gmail.com
}

\begin{abstract}
Introduction: Thyroid disorders are the commonest endocrine disorders affecting women of reproductive age group. The most frequent thyroid disorder in pregnancy is maternal hypothyroidism. Antenatal period can be viewed as a state in which a combination of events concurs to modify the thyroidal economy. Thyroid dysfunction has varied impact on pregnancy outcome. In this study we have assessed the prevalence and the impact of thyroid disorders among antenatal woman registered in the Urban Health Training Centre of a Tertiary care teaching hospital in Kerala, Southern India.

Materials and Methods: A prospective observational study was done among 451 antenatal women registered in the Urban Health Training centre of a Tertiary care hospital. Prevalence of thyroid disorders was assessed based on TSH, Free T4 and Anti TPO antibody status. Maternal and Foetal outcomes with respect to their Thyroid function status were also evaluated.

Results: The prevalence of thyroid dysfunction in pregnancy was $22.39 \%$, subclinical hypothyroidism (20.63\%) was the most common thyroid disorder among these women. Statistically significant association was found between thyroid disorders and maternal complications $(\mathrm{P}$ value $=<0.0000001)$, were as no such significant association was found with regards to foetal outcome $(\mathrm{P}$ value=0.5) and antenatal thyroid dysfunction.

Conclusions: This study concludes that there is a high prevalence of thyroid disorder in pregnancy. Subclinical hypothyroidism was found to be the major thyroid disorder as per this study.
\end{abstract}

Keywords: Antenatal thyroid disorder, Pregnancy thyroid dysfunction, Maternal complications, Foetal complications.

\section{Introduction}

Thyroid disorders constitute one of the most common endocrine disorders in pregnancy. ${ }^{1}$ It is now well established that not only overt, but subclinical thyroid dysfunction also has adverse effects on maternal and foetal outcome. Thyroid dysfunction may be overlooked in pregnancy because of the nonspecific symptoms and hyper metabolic state of normal pregnancy. Pregnancy is associated with profound modifications in the regulation of thyroid function. These changes are the result of various factors like an increase of thyroxin-binding globulin (TBG) due to elevated oestrogen and human chorionic gonadotropin (hCG), increased renal losses of iodine due to increased glomerular filtration rate, modifications in the peripheral metabolism of maternal thyroid hormones, and modification in iodine transfer to the placenta. The physiological changes of pregnancy can simulate thyroid disease. Symptoms of heat intolerance, sluggishness, fatigue, constipation and examination findings of tachycardia, edema, and wide pulse pressure are common to pregnancy and thyroid disease much in same way. ${ }^{2}$ Studies among antenatal women for thyroid dysfunction show prevalence varying from $10 \%-36 \% .^{3-8}$ Women with thyroid dysfunction, both overt and subclinical are at increased risk of pregnancy-related complications such as threatened abortion, preeclampsia, preterm labour, placental abruption, and postpartum haemorrhage. Foetal complications include low-birth-weight babies, first trimester spontaneous abortions, preterm delivery, foetal or neonatal hyperthyroidism, intrauterine growth retardation, high rates of still birth and neonatal deaths, neonatal hyperbilirubinemia, higher incidence of neonatal hypothyroidism, and increased perinatal mortality. ${ }^{9-10}$ In view of the potential adverse outcomes associated with maternal thyroid disorders and the obvious benefits of treatment, some expert panels have suggested routine thyroid function screening in all pregnant women. ${ }^{11}$ In India National guidelines do not recommend routine screening of antenatal women for thyroid dysfunction but it has been suggested in high risk women. These risks include women residing in moderate to high prevalent areas for thyroid disorders, $\mathrm{BMI}>30$ (pre pregnant), Previous/family history of thyroid dysfunction, adverse foetal and maternal outcomes in previous pregnancy, infertility, autoimmune disorders etc. ${ }^{12}$ In view of this existing guidelines to the approach to an antenatal woman with respect to her thyroid status, area specific prevalence of this condition is an important parameter for early diagnosis and treatment of the condition. Most studies done in Southern India have assessed the prevalence of thyroid disorder among pregnant women, but only few have attempted to assess the impact of Thyroid dysfunction on pregnancy. This might be due to the fact that addressing of confounding factors and ethical issues remains a challenge. In this study we have assessed the prevalence and the impact of thyroid disorders among antenatal woman registered in the Urban Health training centre of a Tertiary care teaching hospital in Kerala, Southern India.

\section{Objectives}

1. Primary- To study the prevalence of thyroid dysfunction in antenatal women registered in Urban health Training centre, Ambalapuzha. 
2. Secondary- To assess the impact of thyroid disorders in maternal and foetal outcome.

\section{Materials and Methods}

A prospective observational study was conducted among antenatal women registered under uban health training centre Ambalapuzha, which is a block Primary Health Centre (PHC) attached to the Government TD Medical College Alappuzha, Kerala. This PHC caters to the patients of Ambalapuzha North and South Panchayaths. The sample size was calculated using the formula $4 \mathrm{pq} / \mathrm{d}^{2}$. P was taken as 19.4 (Murthy Nabhi VR. et $\mathrm{al}^{4}$ ) and a relative precision of 4 was substituted in the given formula, thus yielding a sample size of 390. Since we wanted to include all the antenatal women consenting to participate in the study during the study period (1 year), the ultimate number of study subjects included were 451, which was the total number of women (who consented for the study) registered under the unit. Estimation of serum Thyroid Stimulating Hormone (TSH) along with the routine blood investigations were done in all consenting antenatal women with a gestational period of less than 20 weeks. Antenatal women with low or high Ser TSH were further subjected to blood test to assess the Free T4 levels (FT4) and Anti TPO antibody positivity

(Indicator of autoimmunity).

These women were categorised to the following groups based on their Ser TSH, FT4 and Anti TPO Antibodies. ${ }^{4,12,13}$ Group 1- Normal TSH -Euthyroid.

Group 2- High Ser TSH and normal FT4 - Subclinical hypothyroidism.

Group 3- High Ser TSH and low FT4- Overt hypothyroidism.

Group 4- Low Ser TSH and normal FT4 - Subclinical hyperthyroidism.

Group 5- Low Ser TSH and high FT4 - Overt hyperthyroidism.
Group 2 to 5 were further sub classified based on Anti TPO positive /negative status. Women with abnormal Thyroid function test values were referred to a physician for treatment of the same. All the study subjects were followed up periodically (every month till the termination of pregnancy, to assess the various maternal and foetal outcomes.

\section{Results}

A total of 451 antenatal women were included in this study. Mean age of the study subjects were 25.11 years $(\mathrm{SD}=+/$ 4.39).Among the total study subjects, 177 (39.24\%) were from low-socio economic background. Family history of thyroid disorder was found in $35(7.76 \%)$ of study subjects. Thyroid disorders were found among 101 (22.39\%) study subjects. (Table 1) depicts the various thyroid dysfunction among the antenatal women. Only $10(9.90 \%)$ antenatal women with thyroid disorder had anti TPO antibody positive, of which 2 had subclinical hyperthyroidism. Spontaneous miscarriage occurred in 37 women (8.20\%).The mode of delivery was LSCS in $43(10.38 \%)$ antenatal women for various maternal and foetal complications. Thyroid disorder was prevalent in 16 (3.86\%) women subjected to LSCS. Various maternal complications were found in 139 antenatal women, 78 of these women were found to have thyroid dysfunction. Chi square test (Table 2) was done to analyze the difference between the proportion of maternal complication among those with thyroid dysfunction and with normal thyroid function. This was found to be statistically significant ( $\mathrm{P}$ value $=<0.0000001)$. Foetal complications were found in a total of $75(18.11 \%)$ study subjects. Only $19(18.81 \%)$ among the maternal thyroid dysfunction group had foetal complications. On applying the chi square test no statistically significant difference ( $\mathrm{p}$ value $=0.5$ ) was found between the normal maternal thyroid function group and the abnormal maternal thyroid function group with regards to foetal complications (Table 3).

Table 1: Percentage of various thyroid disorders in the study subjects

\begin{tabular}{|l|c|c|}
\hline \multicolumn{1}{|c|}{ Type } & Number & Percentage \\
\hline Euthyroid & 350 & 77.60 \\
\hline Overt Hypothyroidism & 3 & 0.66 \\
\hline Subclinical hypothyroidism & 93 & 20.63 \\
\hline Subclinical Hyperthyroidism & 5 & 1.11 \\
\hline Total & 451 & 100 \\
\hline
\end{tabular}

Table 2: Maternal complications among the study subjects

\begin{tabular}{|l|c|c|}
\hline \multicolumn{1}{|c|}{ Maternal Complications } & $\begin{array}{c}\text { Mother's with abnormal } \\
\text { Thyroid Function(n=101) }\end{array}$ & $\begin{array}{c}\text { Mother's with normal thyroid } \\
\text { function(n=350) }\end{array}$ \\
\hline Anaemia & 43 & 43 \\
\hline Miscarriage & 25 & 12 \\
\hline Pre-eclampsia & 5 & 1 \\
\hline Gestational Diabetes & 4 & 3 \\
\hline Abruption Placenta & 0 & 1 \\
\hline Post partum Haemorrhage & 1 & 1 \\
\hline Total & 78 & 61 \\
\hline
\end{tabular}

$*$ chi square $=131.5 \mathrm{P}$ value $=<0.0000001$

* chi- square test was done taking the total maternal complications among those with thyroid dysfunction and without it. 
Table 3: Foetal complications among the study subjects

\begin{tabular}{|l|c|c|}
\hline \multicolumn{1}{|c|}{ Foetal Complications } & $\begin{array}{c}\text { Mother's with antenatal } \\
\text { Thyroid disorder }(\mathbf{n = 1 0 1})\end{array}$ & $\begin{array}{c}\text { Mother's with Normal Thyroid } \\
\text { Function (n=350) }\end{array}$ \\
\hline Still Birth & 1 & 1 \\
\hline Intra uterine growth retardation & 0 & 5 \\
\hline Low birth weight & 2 & 19 \\
\hline Prematurity & 2 & 0 \\
\hline Neonatal thyroid abnormality & 2 & 1 \\
\hline Neonatal Hyperbilirubinemia & 12 & 29 \\
\hline Neonatal Death & 0 & 1 \\
\hline Total & 19 & 56 \\
\hline
\end{tabular}

$*$ Chi square $=0.44,(\mathrm{P}$ value $=0.50)$

*chi-square test was done taking the total foetal complications among those women with thyroid dysfunction and those without it.

\section{Discussion}

This study was an attempt to assess the scenario of thyroid dysfunction and its impact on pregnancy outcome. In view of the inconsistent results from various studies on this subject done across the globe, such a study is relevant for better understanding of the situation locally. Studies among antenatal women for thyroid dysfunction show prevalence varying from $10 \%-36 \% .^{3-8}$ The prevalence of thyroid dysfunction $(22.39 \%)$ in this study is more in line with the results of a study by Raj put $\mathrm{R}$ et al in Harayana. ${ }^{14}$ Though the national guidelines do not recommend universal screening of pregnant women for thyroid disorder, In Kerala, it is a routine practice to screen the women for thyroid disorder with baseline serum TSH assessment during the early period of gestation. If the TSH values are deranged, Free T4 and Anti TPO antibody determination is a mandatory practice. This protocol helps in identification of thyroid dysfunction early in pregnancy, following which corrective measures are taken at an early stage which prevents maternal and fetal complications. In this study main thyroid disorder was subclinical hypothyroidism (20.63\%) this finding is similar to the results of various other studies done across India. ${ }^{4,14}$ There are various studies which sheds light on the facts related to maternal and foetal complications as a result of thyroid dysfunction. The various complications which might occur due to this disorder ranges from miscarriage in pregnant women to foetal deaths. The gamut of complications warrants early identification and prompt correction of this condition..$^{13}$ In this study we got a statistically significant association between maternal complications and thyroid disorder in pregnancy, the complication studied were anaemia, miscarriage, pre-eclampsia, gestational diabetes mellitus, abruption placenta and post partum haemorrhage. In a study by Vimal Nambiar et al, women with thyroid disorder had 3 times more miscarriage when compared to the normal (thyroid status) antenatal women, rest of the outcomes were similar in both the groups. ${ }^{15}$ Pre eclampsia was found to have statistically significant association with hypothyroidism as per a study by Kharb S. ${ }^{16}$ However we did not get a statistically significant association between fetal outcomes and thyroid status of these women. Though there are various studies which show that thyroid disorders might result in various fetal complications like still birth, IUGR, low birth weight, neonatal hypothyroidism, neonatal hyper bilirubinemia and neonatal death. ${ }^{9-10}$ This study failed to establish a statistically significant association between maternal thyroid disorder and foetal complications. There are few studies with similar findings as that of the present study, notable among them is the study by Bluementhal NJ, which is a cohort study done in Mumbai, the findings revealed that there was no significant difference between documented complications of pregnancy outcomes in the group with thyroid disorder and euthyroid pregnant women. ${ }^{17}$ All the antenatal women in this study were referred to a physician, if diagnosed with thyroid dysfunction and were followed up by the investigators for compliance of medications (as prescribed by the physician). Most of the treated antenatal women had near normal thyroid function test values during their follow up period. Early diagnosis and prompt treatment might be the reason for overall rate of low maternal and foetal complications in the study group. Ethical issues and confounding factors like thyroid medications are a hindrance to studies of similar nature. Revised guidelines by the American Thyroid association ${ }^{13}$ emphasis the need for a regional data base for determining the upper threshold of thyroid hormone values in pregnancy. There is also a need for revising the existing national guidelines for the identification of thyroid disorders in pregnancy.

\section{Conclusion}

This study concludes that there is a high prevalence of thyroid disorder in pregnancy $(22.39 \%)$. Subclinical hypothyroidism is the most prevalent among them. The study also highlights a significant association between maternal thyroid dysfunction and maternal complications. There was no statistically significant association between antenatal thyroid disorders and foetal complications. Large scale population based studies addressing the confounding factors to assess the impact of thyroid dysfunction on pregnancy outcomes is called for. 


\section{Source of Funding}

None.

\section{Conflict of Interest}

None.

\section{References}

1. Abalovich M, Amino N, Barbour LA, Cobin R H, De Groot LJ, Glinoer D, et al. Management of thyroid dysfunction during pregnancy and postpartum: an endocrine society clinical practice guideline. J Clin Endocrinol Metab. 2007; 92(8):1-47.

2. Sharma PP, Mukhopadhyay P, Mukhopadhyay A,

Muraleedharan PD, Beegum N. Hypothyroidism in pregnancy. J Obstet Gynecol India. 2007;57:331-4.

3. Stagnaro-Green A. Overt hyperthyroidism and hypothyroidism during pregnancy. Clin Obstet Gynecol. 2011;54(3):478-87

4. Murthy Nabhi VR. Uma B,JmR,Sampurna K,Vasantha K, Vijayalakshmi G. High prevalence of subclinical hypothyroidism in pregnant women in South India. Int $J$ Reprod Contracept Obstet Gynecol. 2015;4(2):453-6.

5. Pahwa S, Mangat S. Prevalence of thyroid disorders in pregnancy. Int J Reprod Contracept Obstet Gynecol. 2018;7(9):3493-6.

6. Sahu MT, Das V, Mittal S, Agarwal A, Sahu M. Overt and subclinical thyroid dysfunction among Indian pregnant women and its effect on maternal and fetal outcome. Arch Gynecol Obstet. 2010;281(2):215-20.

7. Dhanwal DK, Bajaj S, Rajput R, Subramaniam KAV, Chowdhry S, Bhandari R, et al. Prevalence of hypothyroidism in pregnancy: An epidemiological study from 11 cities in 9 states of India. Indian J Endocrinol Metab. 2016;20(3):387-90.

8. Pillai NS, Bennet J. Prevalence of hypothyroidism amongst pregnant women: a study done in rural set up. Int $J$ Reprod Contraception, Obstet Gynecol. 2018;7(4):1586.

9. LeBeau SO, Mandel SJ. Thyroid disorders during Pregnancy. Endocrinol Metab Clin North Am. 2006;35(1):117-36.
10. Casey B, Leveno K. Thyroid disease in pregnancy. Obstet Gynecol. 2006;108(5):1283-92.

11. Surks MI. Commentary: Subclinical thyroid dysfunction: A joint statement on management from the American Association of Clinical Endocrinologists, the American Thyroid Association, and the Endocrine Society. J Clin Endocrinol Metab. 2005;90(1):586-7.

12. Welfare ministry of health and family. National guidelines for screening of hypothyroidism during pregnancy. Matern Heal Div Minist Heal Fam Welfare, Gov India. 2014;(December):148.

13. Alexander EK, Pearce EN, Brent GA, Brown RS, Chen H, Dosiou C, et al. 2017 Guidelines of the American Thyroid Association for the Diagnosis and Management of Thyroid Disease during Pregnancy and the Postpartum. Thyroid. 2017;27(3):315-89.

14. Rajput R, Goel V, Na Indian J Endocrinol Metab. 2015;19(3):416-9.

15. Nambiar V, Jagtap VS, Sarathi V, Lila AR, Kamalanathan S, Bandgar TR, et al. Prevalence and impact of thyroid disorders on maternal outcome in Asian-Indian pregnant women. $J$ Thyroid Res. 2011; Article ID 429097. doi. org/10.4061/2011/429097

16. Kharb S, Sardana D, Nanda S. Correlation of thyroid functions with severity and outcome of pregnancy. Ann Med Health Sci Res. 2013;3(1):43-6.

17. Blumenthal NJ, Eastman CJ. Beneficial Effects on Pregnancy Outcomes of Thyroid Hormone Replacement for Subclinical Hypothyroidism. J Thyroid Res. 2017; Article ID 4601365. doi.org/10.1155/2017/4601365

How to cite this article: Ramachandran R, Mohan L, Jose MS. Prevalence of thyroid disorders in antenatal women and its impact on maternal and foetal outcome. Indian $J$ Forensic Community Med. 2020;7(1):29-32. 